\title{
THE ULTRAVIOLET SPECTRA OF RADIO-LOUD AND RADIO-QUIET QUASARS ${ }^{1}$
}

\author{
Paul J. Francis, ERIC J. HoOPer, AND Chris D. IMPey \\ Steward Observatory, University of Arizona, Tucson, Arizona 85721 \\ Electronic mail: pjf@as.arizona.edu, ehooper@as.arizona.edu, impey@as.arizona.edu \\ Received 1993 March 10; revised 1993 April 21
}

\begin{abstract}
The rest-frame ultraviolet spectral properties of matched samples of radio-loud, radio-moderate, and radio-quiet quasars are investigated, using quasars drawn from the Large Bright QSO Survey. We confirm the absence of spectral differences between radio-loud and radio-quiet quasars at rest-frame wavelengths longward of $1600 \AA$, as reported by previous authors. However, at shorter wavelengths we find a significant difference: radio-loud quasars have narrower (96\% confidence), higher equivalent-width (97\% confidence) Lyman- $\alpha$ and $\mathrm{C}$ IV emission lines. We further investigate quasars which are radio quiet, but have radio-to-optical flux ratios at the upper extreme of the radio-quiet population. Broad absorption line quasars are overabundant by a factor of $\sim 10$ in this radio-moderate population; the overabundance is significant at a $99.99 \%$ confidence level.
\end{abstract}

\section{INTRODUCTION}

The dichotomy in the radio properties of quasars is one of the oldest and most enduring problems of the field. The ratio of the radio to optical power of quasars spans more than four orders of magnitude, and is clearly bimodal (Kellerman et al. 1989; Visnovsky et al. 1992; Hooper et al. 1993; Stocke et al. 1992). Radio-loud quasars may have an excess of associated absorption lines (Foltz et al. 1986) and strong, flat spectrum x-ray emission (Lawson et al. 1992).

Despite the great differences at radio wavelengths, the optical spectra of radio-loud and radio-quiet quasars are remarkably similar. Boroson \& Green (1992) find that low redshift radio-loud quasars tend to have strong [O III] emission and weak optical Fe II emission compared to the radio-quiet quasars, but neither Steidel \& Sargent (1991) nor Corbin (1992) find any compelling spectral differences in higher redshift quasars. Baldwin et al. (1989) identify possible differences, but they are unable to distinguish between trends with radio power and with optical luminosity.

In this paper we investigate the rest-frame UV spectra of well matched samples of radio-loud, radio-moderate, and radio-quiet quasars, all drawn from a homogeneous optically selected quasi-stellar object (QSO) sample, the Large Bright QSO Survey (LBQS). This paper probes shorter rest-frame wavelengths, and lower radio-to-optical flux ratios, than any existing work. The survey and observations are described in Sec. 2, the analysis techniques and results in Sec. 3, and the results are discussed in Sec. 4.

\section{OBSERVATIONS}

All the quasars analyzed in this paper are drawn from a single optically selected sample, the LBQS. This ensures

${ }^{1}$ Observations reported here were obtained with the Multiple Mirror Telescope, a facility jointly operated by the Smithsonian Institution and the University of Arizona, and with the Las Campanas Observatory, a facility of the Carnegie Institution of Washington. that they are well matched in redshift and luminosity, and that the spectral observations are of uniform quality. The LBQS consists of data on 1018 quasars, with redshifts $0.2 \leqslant z \leqslant 3.4$ and apparent photographic $B_{J}$ - band magnitudes $16.0 \leqslant m_{B_{J}} \leqslant 18.85$. A description of the survey selection, the observing procedures and plots of all the spectra can be found in Foltz et al. $(1987,1989)$, Hewett et al. (1991), Chaffee et al. (1991), and Morris et al. (1991). The quasars are selected from objective prism plates using a variety of criteria, and the survey has proven to be sensitive to all known types of quasars, with the possible exception of red BL Lac objects. Comparisons with surveys selected using different techniques and at different wavelengths suggest that the LBQS is highly complete within its redshift range.

LBQS spectra were obtained using the Multiple Mirror Telescope, whose data provide a spectral coverage of 32007400 at $6 \AA$ resolution, and with the Du Pont Telescope of the Las Campanas Observatory (3200-6800 $\AA$ at $10 \AA$ resolution). Each quasar was observed until a signal-tonoise ratio of $\sim 10$ was obtained in the rest-frame wavelength region nearest to $4000 \AA$ in the observed frame. Blue magnitudes accurate to $0.15 \mathrm{mag}$ were obtained by automated scans of UK Schmidt direct plates, calibrated by CCD imaging. Note that due to the small apertures used in the MMT observations, the large-scale continuum energy distributions of some of the spectra are unreliable, and thus no attempt has been made to investigate continuum properties in this paper.

255 optically bright quasars were selected for radio observation. The sub-sample was chosen to cover the whole range of LBQS redshifts, without reference to the spectral properties. Observations were made with the Very Large Array $^{2}$ (VLA) at a frequency of $8.4 \mathrm{GHz}$, using the $\mathrm{C}$ and $\mathrm{C} / \mathrm{D}$ array configurations, and the integration times

\footnotetext{
${ }^{2}$ The Very Large Array (VLA) of the National Radio Astronomy Observatory is operated by Associated Universities, Inc., under a cooperative agreement with the National Science Foundation.
} 
TABLE 1. LBQS quasars from the MG catalog

\begin{tabular}{lll}
\hline \hline & & \\
LBQS Name & MG Name & Other Name \\
\hline & & \\
$0056+0009$ & $0059+0006$ & PHL 923 \\
$0100-2702$ & $\ldots$ & PKS 0100-270 \\
$0106+0119$ & $0108+0134$ & PKS 0106+01 \\
$1012+0213$ & $1015+0158$ & PKS 1012+022 \\
$1130+0032$ & $1133+0015$ & $\ldots$ \\
$1137+0110$ & $1140+0053$ & $\ldots$ \\
$1210+1324$ & $1213+1307$ & DW 1211+13 \\
$1215+1121$ & $1218+1104$ & MC 1215+113 \\
$1218+1734$ & $1220+1718$ & $\ldots$ \\
$1226+1035$ & $1228+1018$ & MC $1226+105$ \\
& & \\
\hline \hline
\end{tabular}

ranged from 7 to $11 \mathrm{~min}$. A full account of the radio observations can be found in Visnovsky et al. (1992) and Hooper et al. (1993).

Radio maps were processed with a point-source detection algorithm; a quasar was listed as detected if the measured flux exceeded three times the standard deviation of the noise, and if the positional agreement between the radio and optical positions was better than 2 arcsec. Given this $3 \sigma$ flux limit, $\sim 2$ spurious detections are expected. The expected number of chance positional coincidences is less than 0.05 in the whole sample (Visnovsky et al. 1992). Fluxes include only the component unresolved on a scale of $\sim 3$ arcsec, however, fewer than $20 \%$ of the detections have discernible extended emission, and in no case does the extended flux exceed $50 \%$ of the core flux. Radio-tooptical flux ratios are constructed between the rest-frame Johnson $B$ band and rest-frame $8.4 \mathrm{GHz}$. Optical $k$ corrections were computed from a LBQS composite spectrum (Francis et al. 1991), and radio $k$ corrections assumed a spectral index $\alpha=-0.5\left(f_{v} \propto v^{\alpha}\right)$.

In order to enlarge the sample of radio-loud quasars, the remainder of the LBQS not observed with the VLA was checked for positional coincidences with the $5 \mathrm{GHz}$ MITGreenbank (MG) survey (Bennett et al. 1986). Ten coincidences to within two standard deviations of the radio positional accuracy were found; 0.36 would be expected by chance. One additional LBQS quasar was identified as radio loud by positional coincidence with a Parkes radio source listed by Veron-Cetty \& Veron (1987). These additional detections (with the exception of one which was also detected with the VLA) are listed in Table 1.

\section{RESULTS}

45 quasars $(18 \%)$ were detected by the VLA measurements. The distribution of quasars as a function of optical luminosity and redshift is discussed by Hooper et al. (1993); for the purposes of this paper we consider only radio-to-optical flux ratios. The redshift and luminosity distributions of detected and undetected sources do not differ significantly.

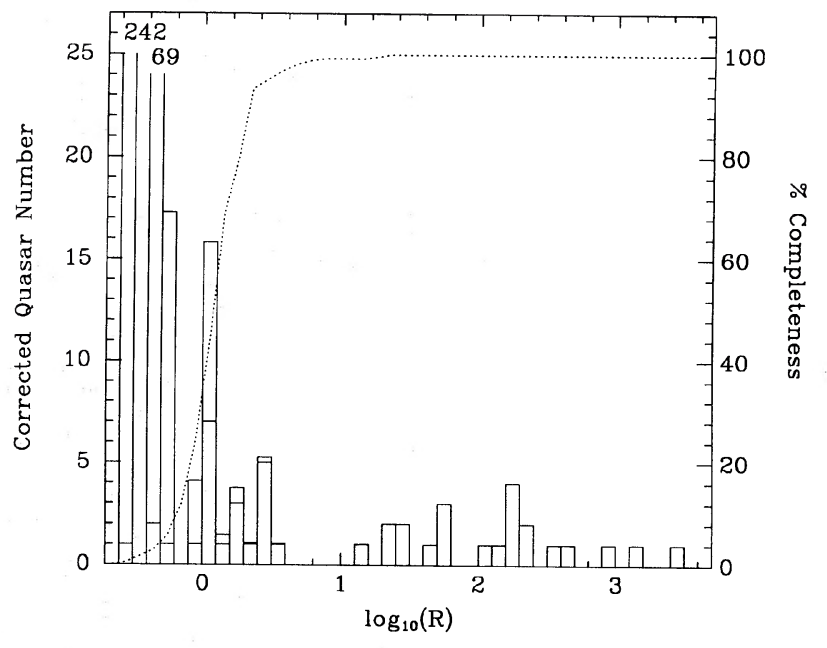

FIG. 1. Distribution of radio-to-optical flux ratios $R$ for the quasars with VLA observations. The histogram shows the distribution of the quasars in $R$, corrected for the sample completeness as described in the text (lefthand axis). The dotted line shows the percentage completeness of the survey as a function of $R$ (right-hand axis). The uncorrected number of quasars is shown as the lower bars in the histogram columns; the difference between corrected and uncorrected distributions is invisible for $\log _{10}(R)>0.5$.

Despite the large fraction of the quasars with only upper bounds on their radio fluxes, limits can be placed on the distribution of the radio-to-optical flux ratios $R$. The method is this; due to the uneven sensitivity of the observations and the range of optical luminosities, a small number of the observations are sensitive to quasars with radioto-optical flux ratios as small as $\log _{10}(R)=-0.5$, whereas most are sensitive only to quasars with radio-to-optical flux ratios greater than $\log _{10}(R)>0.1$. Using the distribution of the limiting radio-to-optical ratios, the fraction of the quasars which would have been detected if they had had a given radio-to-optical ratio can be computed. This completeness fraction is then used to correct the number of detected quasars in each bin of radio-to-optical flux, yielding an estimate of the true distribution of radio-to-optical fluxes. For example, three quasars are observed to have $-0.3>\log _{10}(R)>-0.4$, while only $3 \%$ of the observations are sensitive to $R$ values this low. Thus if the whole sample had been sensitive to such small $R$ values, $\sim 3 / 0.03$ $=100$ would have been seen. Note that sidelobes in the VLA maps containing strong sources make accurate error determination impossible. The completeness fraction has thus been computed using only the 242 observations in which no object with $R>100$ was detected. As nothing was known a priori about the radio properties of the quasars, the noise properties of the observations with strong detections will not greatly differ from the rest.

This corrected distribution of radio-to-optical fluxes $R$ is shown in Fig. 1, along with the uncorrected distribution and the completeness fraction. The distribution is reasonably well defined for $\log _{10}(R) \gtrsim 0$ and two populations are clearly distinguished, a radio-loud population with $\log _{10}(R)>1.2$ and a radio-quiet population with $\log _{10}(R)$ $<0.7$. This well-known bimodality occurs at the same 
value of the radio-to-optical flux ratio as reported by other authors (e.g., Kellerman 1989; Stocke et al. 1992).

None of our observations probe radio-to-optical flux ratios below $\log _{10}(R)=-0.6$, and so the shape of the distribution at these low values is undefined. However, a large fraction of the few observations going as deep as $\log _{10}(R) \sim-0.5$ are detections. For example, three of the seven quasars observed to $\log _{10}(R)<-0.3$ were detected, and the one quasar observed to $\log _{10}(R)<-0.5$ was detected. This suggests that over half of the survey quasars may have $-0.6<\log _{10}(R)<-0.3$. If this is the case, few quasars remain to have $\log _{10}(R)<-0.6$, though those few could have extremely low values of $R$.

For the purposes of this paper, we define three quasar sub-samples. Radio-loud quasars are those with $\log _{10}(R)>1.0$, radio quiet have $\log _{10}(R)<0.2$ with radiomoderate quasars have $0.2<\log _{10}(R)<1.0$. The value of $\log _{10}(R)=0.2$ for the divide between radio-moderate and radio-quiet quasars is chosen because the radio detections are $75 \%$ complete above this value; the results reported in this paper are insensitive to the exact value selected. The two quasars with upper limits above $\log _{10}(R)=1.0$ are not used, and the 44 quasars with upper limits in the range $0.2<\log _{10}(R)<1.0$ are classed as radio quiet, though it is expected that $\sim 4$ will actually be radio moderate.

\subsection{Broad Absorption Lines}

The one well-established relation between radio properties and optical spectra is the absence of radio-loud broad absorption line quasars (BALQSOs, e.g., Stocke et al. 1992). Given the limited signal-to-noise ratio and resolution of our spectra, it is not always possible to unambiguously decide if a given quasar is a BALQSO. Luckily, Weymann et al. (1991, hereafter referred to as PALBAL) obtained high quality optical spectra of 23 LBQS BALQSOs with redshifts above 1.5 as a part of their study of the spectral properties of BAL and non-BAL quasars. They applied a consistent technique to decide if their objects were BALQSOs, and we adopt their list. Ten of our objects are listed as definite BALQSOs by their criteria, of which one $(1235+1807 \mathrm{~B})$ is a low-redshift $\mathrm{Mg}$ II BALQSO.

None of our 12 radio-loud quasars with redshifts above 1.5 are listed as BALQSOs in PALBAL. Indeed, a visual inspection of the spectra reveals no definite or possible BAL features in any of the radio-loud quasars regardless of their redshift. Our data are thus consistent with the absence of radio-loud BALQSOs.

A very different picture emerges amongst the radiomoderate quasars. Of the six radio-moderate quasars with redshifts greater than 1.5, four are listed as BALQSOs in PALBAL. Only nine of the 111 quasars with detections or upper limits in the range $\log _{10}(R)<1.0$ (i.e., radio moderate or radio quiet) and redshifts above 1.5 are listed as BALQSOs by PALBAL. Given this frequency of BALQSOs, the binomial probability of finding four or more BALQSOs in any randomly selected sample of six quasars is $6 \times 10^{-4}$. We conclude that the fraction of radio- moderate quasars with BAL features is significantly higher than in the radio-quiet population, confirming a tentative suggestion of Stocke et al. (1992). The mean absolute magnitudes of the radio-moderate and radio-quiet samples differ by only 0.03 mag., so luminosity differences should not affect this conclusion. Note also that the low-ionization BALQSO $1235+1807 \mathrm{~B}$ has a radio-to-optical flux ratio $\log _{10}(R)=0.5$, making it one of the strongest radio emitters of the radio-moderate population.

The above analysis relies upon selecting a somewhat arbitrary dividing line between radio-moderate and radioquiet quasars, and makes no use of the information contained in the upper limits. To check this result, we therefore used a survival analysis technique: the Gehan Statistic. The Gehan Statistic (Feigelson \& Nelson 1985) is a generalization of the nonparametric Wilcoxon test statistic to data with upper limits. We excluded radio-loud quasars from the analysis. The two-sided probability that the BALQSO and non-BALQSO samples have $R$ values drawn from the same distribution was $1.2 \times 10^{-7}$. If the alternative Log-Rank weighting scheme is used, the probability remains very low; $1.8 \times 10^{-5}$. We conclude that the excess radio-to-optical ratios of the BALQSOs are not an artifact of the definition of "radio moderate," and that including upper-limit information enhances the significance of this result.

Are any of the BALQSOs definitely radio quiet? Three of the PALBAL BALQSOs have upper limits $\log _{10}(R)<0.2$ and one has $\log _{10}(R)<0.0$, so at least some radio-quiet quasars are BALQSOs.

Could the preponderance of BALQSOs in the radiomoderate population be simply due to their faintness in the optical caused by the absorption troughs? The BAL absorption will depress the optical magnitude and thus enhance the ratio $R$. The size of this effect was estimated by interactively measuring the equivalent widths of all BAL troughs lying within the $B_{J}$ passband, in the radiomoderate BALQSOs. The BAL troughs reduced the $B_{J}$ flux by between $6 \%$ and $40 \%$ with a median loss of $16 \%$, which as Fig. 1 shows is too little to generate the radiomoderate population by enhancing the $R$ values of radioquiet quasars. Note however that if $\mathrm{Mg}$ II BALQSOs are dusty, as suggested by Sprayberry \& Foltz (1992), their optical fluxes could be greatly suppressed, leading to the high observed $R$. An extinction factor of $\sim 5$ at $1500 \AA$ would suffice to put most $\mathrm{Mg}$ II BALQSOs in the radiomoderate population, even if their intrinsic spectra were identical to those of radio-quiet quasars.

The sample is too small to test for differences in the strength and shape of the BAL troughs between radiomoderate and radio-quiet quasars, but we note in passing that the mean "balnicity index" as defined by PALBAL is higher in radio-moderate BALs, but that they have a smaller "detachment index." Neither difference is statistically significant at the $2 \sigma$ level.

\subsection{Emission-Line Properties}

The measurement of emission-line parameters is a notoriously subjective process (Francis 1991), and for this 
reason we compared the emission-line properties of radioloud and radio-quiet quasars in three distinct ways: by forming composite spectra, by direct measurement, and by principal components analysis (PCA).

\subsubsection{Composite spectra}

Composite spectra of the radio-loud and radio-quiet populations were constructed as a first look at the data. BALQSOs were excluded from the composites; this meant that too few radio-moderate quasars remained to give a meaningful comparison, so they were combined with the radio-quiet population. Using redshifts derived from crosscorrelation with the composite spectrum of Francis et al. (1991), each spectrum was shifted to its rest frame and rebinned on a uniform wavelength scale. For each of three rest-frame wavelength regions, all the spectra showing the entirety of that region were normalized to have unit flux over that wavelength region and were summed. The variance of the spectra summed to give each bin was also recorded.

The rest-frame wavelength regions used were: 1150 $1650 \AA$ (the 79 radio-quiet and 8 radio-loud quasars with $3.5>z>1.8$ contributed), $1700-2150 \AA$ (the 124 radioquiet and 21 radio-loud quasars with $2.44>z>0.9$ contributed), and $1900-3050 \AA$ (the 53 radio-quiet and 14 radioloud quasars with $1.4>z>0.7$ contributed). All results were checked by constructing composites with narrower rest-frame wavelength coverages (centered on emission lines) and hence more contributing spectra. In no case did these narrow-coverage composites differ appreciably from the wide wavelength-range composites.

The composites are shown in Fig. 2. Differences in continuum slope may be artifacts of the narrow apertures and large airmasses of certain observations. No difference between the radio-loud and quiet quasars is seen at the wavelengths of $\mathrm{Mg}$ II and $\mathrm{C}$ III]. There is possibly a small difference at around $2300 \AA$ in a blend of Fe II emission (Wills et al. 1985), N v may be slightly stronger in the radio-quiet composite, but both $\mathrm{C}$ IV and Lyman- $\alpha$ are clearly stronger in the radio-loud composite.

Could the excess equivalent-widths of $\mathrm{C} \mathrm{IV}$ and Lyman- $\alpha$ be due to the Baldwin effect, the well known tendency of brighter quasars to have lower equivalentwidth C IV? The radio-loud quasars with redshifts high enough to see $\mathrm{C}$ IV have an average absolute magnitude 0.1 mag brighter than that of the radio-quiet sample, so the Baldwin effect works in the opposite sense to the correlation noted here.

\subsubsection{Direct parameter measurements}

The composite spectra provide a model-free look at the spectral differences between the classes, but they are insensitive to some line-profile changes and can be badly affected by small numbers of extreme spectra. For this reason, line parameters were measured directly for Lyman- $\alpha, \mathrm{N} \mathrm{v}$, $\mathrm{C}$ IV, and Fe II. BALQSOs were excluded from the measurements. Equivalent widths $W$ were measured as described in Francis (1993) using the same continuum windows and integration limits for Lyman- $\alpha, \mathrm{C}$ IV, C III] and
$\mathrm{Mg}$ II. For $\mathrm{N}$ v, continuum windows were $1150-1180$ and $1250-1300 \AA$, and the integration limits were $1230-1250$ $\AA$; these limits will include substantial flux from Lyman- $\alpha$. For Fe II, continuum windows were 2200-2300 $\AA$ and 2500-2600 $\AA$, and the integration limits were $2300-2500$ $\AA$. Note that the integration limits for Fe II are narrower than conventionally employed; they were chosen to concentrate on the $\mathrm{Fe}$ II blend showing the maximum difference between the radio-loud and quiet composite spectra (Fig. 2). Results are shown in Table 2. In addition, peak heights (the ratio of the line peak flux to the underlying continuum flux) and velocity widths (full width at half maximum height, FWHM) were measured interactively for all the C IV profiles. C IV was chosen as the one relatively unblended line for which a difference was seen in the composite spectra. For C III] the direct parameter measurement showed differences between the mean equivalent widths of the radio-loud and quiet samples, which were not seen in the composite spectra. The differences proved to be strongly dependent on the placing of the continuum windows, and could go in either sense. A small number of radio-loud quasars observed at high airmass and with redshifts such that the emission line appeared at a very blue observed-frame wavelength were responsible for the discrepancy. When these objects were removed, no discrepancy remained, and the composite spectra (Fig. 1) show no features at the wavelengths of these lines. We therefore assign the radio-loud population the same mean equivalent width as is measured for the radio-quiet population.

Table 2 shows that the only differences significant at the $2 \sigma$ level are the equivalent widths of Lyman- $\alpha$ and C IV. To further test the reality of these excesses, a KolmogorovSmirnov (KS) test was carried out on the equivalent-width distributions. This test is insensitive to small numbers of extreme spectra. The two-sided probabilities of obtaining differences this large were $9 \%$ for $\mathrm{C} \mathrm{IV}$ and $7 \%$ for Lyman- $\alpha$, so the equivalent-width excesses are of marginal significance.

The peak heights and velocity widths of $\mathrm{C}$ IV are shown in Fig. 3. Peak heights and widths strongly anticorrelate (Francis et al. 1992), and radio-loud and moderate objects are strongly concentrated toward low velocity widths. The mean velocity width (FWHM) of the 12 contributing radio-loud quasars is $5000 \pm 570 \mathrm{~km} \mathrm{~s}^{-1}$, of the 3 radiomoderate quasars $4500 \pm 950 \mathrm{~km} \mathrm{~s}^{-1}$ and of the 106 radioquiet quasars $6700 \pm 260 \mathrm{~km} \mathrm{~s}^{-1}$. A KS test on the distributions yields a two-sided probability of the FWHM distributions of radio-loud and quiet quasars being drawn from the same population of $4 \%$. The radio-moderate population is too small for the differences to be significant. Note that the one radio-loud quasar with a FWHM greater than the mean of the radio-quiet population is not a measurement error.

Thus the excess line emission of Lyman- $\alpha$ and C IV radio-loud quasars is significant at a $90 \%$ confidence level, and the narrowness of $\mathrm{C}$ IV in radio-loud quasars is significant at a $96 \%$ confidence level, based on these data alone. 

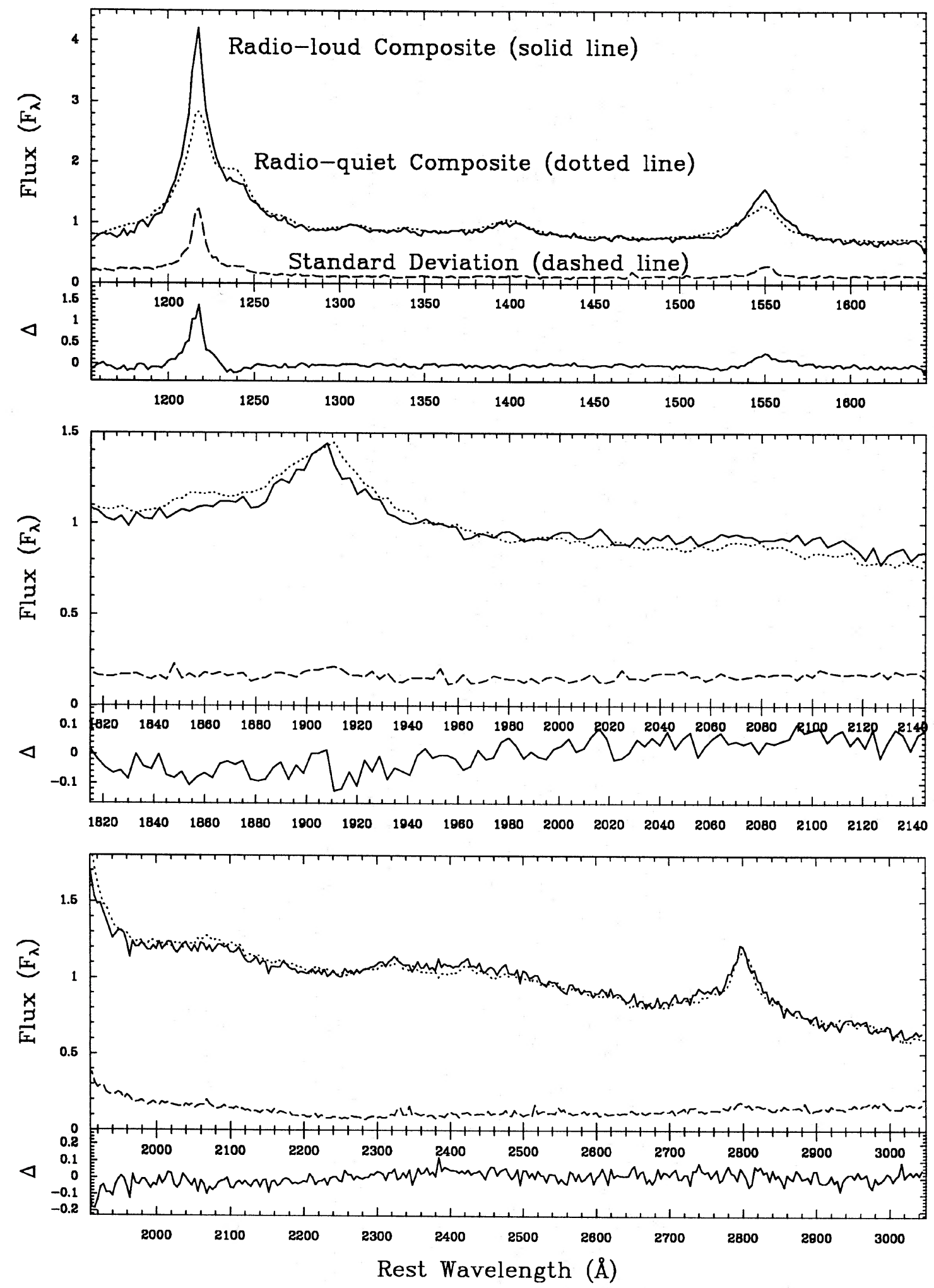

FIG. 2. Composite spectra of the radio-loud and quiet populations. Three different rest-frame wavelength regions are shown. The top panel in each shows the composite radio-loud spectrum (solid line), the composite radio-quiet spectrum (dotted line), and the standard deviation of the radio-quiet spectra (dashed line at the bottom). The bottom panel in each shows the difference $\Delta$ between the radio-loud and the radio-quiet composites (loud minus quiet). 
TABLE 2. Emission-line equivalent widths.

\begin{tabular}{lcccc}
\hline \hline & & & & \\
Line & \multicolumn{2}{c}{ Number } & Equivalent Width W $(\AA)$ \\
& Loud & Quiet & Loud & Quiet \\
\hline & & & & \\
Lyman- $\alpha$ & 7 & 78 & $58 \pm 9.8$ & $37 \pm 2.0$ \\
N V & 7 & 78 & $17 \pm 1.8$ & $20 \pm 0.5$ \\
C IV & 12 & 122 & $28 \pm 3.6$ & $19 \pm 0.7$ \\
C III] & 21 & 124 & $19 \pm 1.4$ & $19 \pm 0.6$ \\
Fe II & 18 & 103 & $15 \pm 1.6$ & $14 \pm 1.4$ \\
Mg II & 18 & 98 & $64 \pm 4.2$ & $67 \pm 2.7$ \\
& & & & \\
\hline \hline
\end{tabular}

\subsubsection{Principal components analysis}

The narrow $\left(\sim 2500 \mathrm{~km} \mathrm{~s}^{-1}\right)$, strongly peaked, high equivalent-width high ionization lines seen in the radioloud population are the defining characteristics of the narrow-line QSO sub-class (NLQSOs, Baldwin et al. 1988; Francis et al. 1992). Indeed the difference spectrum between radio-loud and radio-quiet quasars (Fig. 2) bears a strong resemblance to the principal component used to define the NLQSO class in Francis et al. Can the emissionline differences between radio-loud and radio-quiet quasars be explained simply by an overabundance of NLQSOs in the radio-loud population?

In Francis et al. (1992) the NLQSO class is identified by its strong first component weight in the classification scheme defined in that paper. The mean first component weight of the 8 radio-loud quasars analyzed is $2.36 \pm 1.19$, while that of the 72 radio-quiet quasars is $-0.08 \pm 0.29$.
TABLE 3. Comparison with other authors.

\begin{tabular}{|c|c|c|c|c|}
\hline \multirow[b]{2}{*}{ Line } & \multirow[b]{2}{*}{ LBQS } & \multicolumn{2}{|c|}{ Ratio W(loud)/W(quiet) } & \multirow[b]{2}{*}{ Weighted Mean } \\
\hline & & Corbin & Steidel \& Sargent & \\
\hline C IV & $1.47 \pm 0.24$ & $1.17 \pm 0.27$ & $1.30 \pm 0.21$ & $1.32 \pm 0.14$ \\
\hline C III] & $1.00 \pm 0.11$ & $1.13 \pm 0.28$ & $0.92 \pm 0.10$ & $0.96 \pm 0.07$ \\
\hline $\mathrm{Fe}$ II & $1.06 \pm 0.23$ & $1.27 \pm 0.15$ & $\ldots$ & $1.22 \pm 0.12$ \\
\hline $\mathrm{Mg}$ II & $0.96 \pm 0.12$ & $\ldots$ & $1.06 \pm 0.13$ & $1.01 \pm 0.09$ \\
\hline
\end{tabular}

The difference is thus $2.44 \pm 1.22$, significant at the $2 \sigma$ level. A KS test yields a $6.6 \%$ two-sided probability that the two samples were drawn from the same population. Alternatively, note that of the five most extreme NLQSOs (first component weight $>4$ ), three are radio-loud (and another is radio-moderate), although the radio-quiet sample is nine times bigger than the radio-loud sample. Visual inspection shows that only one of the radio-loud quasars (1323-0248) is definitely not a NLQSO, and two more are borderline cases.

The conclusion is that NLQSOs are overabundant in the radio-loud population. The correspondence between NLQSOs and radio-loud quasars is not, however, perfect.

\subsubsection{Comparison with existing work}

Both Steidel \& Sargent (1991) and Corbin (1992) have analyzed the emission-line properties of C IV, C III], Fe II, and $\mathrm{Mg}$ II in radio-loud and radio-quiet quasars without finding significant differences. Is the discrepancy significant, and what does the combined data-set tell us?

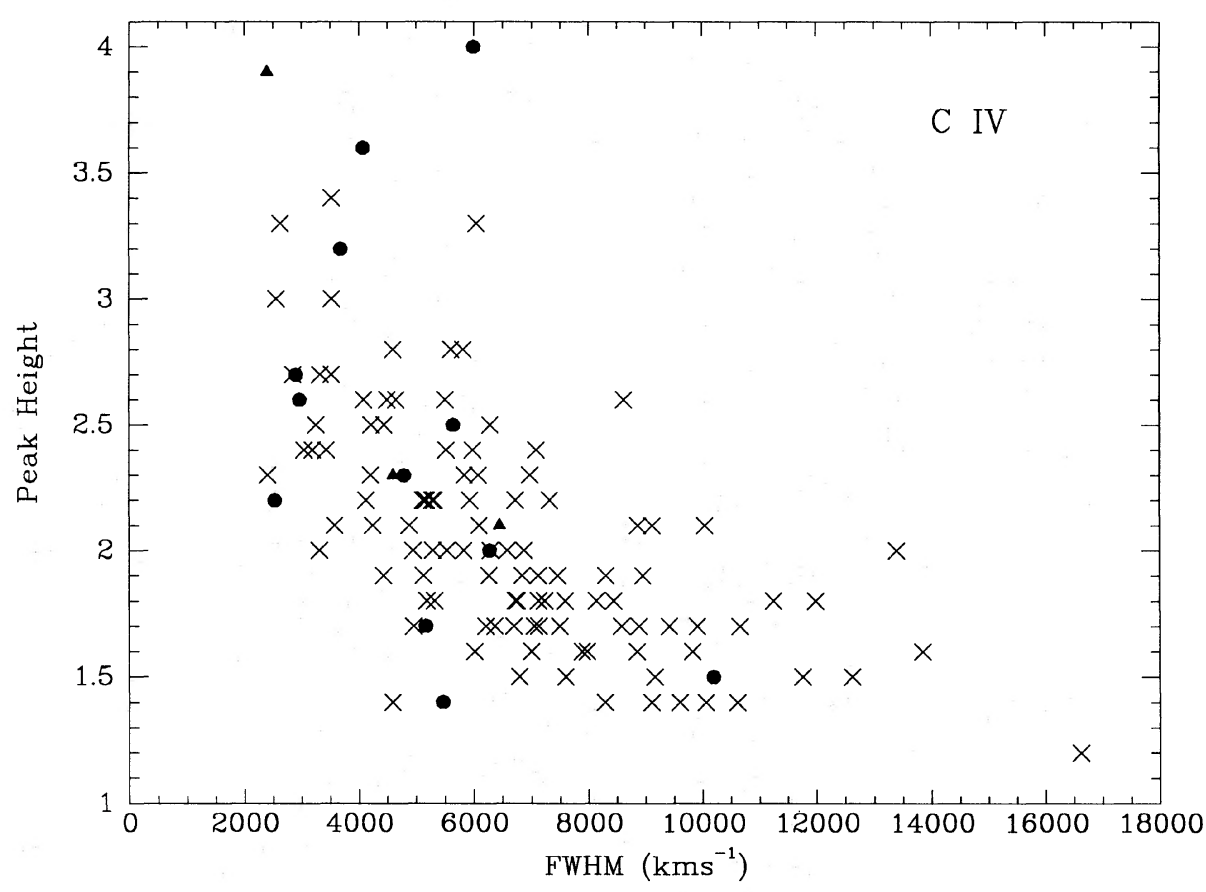

FIG. 3. Peak heights (the ratio of the flux at the line peak to the flux in the underlying continuum) and velocity widths (FWHM) for the C IV emission line of all non-BALQSO quasars with radio observations. Filled circles are radio-loud quasars, filled triangles are radio-moderate quasars and crosses are radio-quiet quasars. 
Table 3 shows equivalent width $(W)$ data compiled from their papers and from this work. As the methods of measuring equivalent widths and the quality of the spectra differ greatly between the surveys, we have not attempted to compare the equivalent widths directly. Instead, we show the ratios of the equivalent widths in the radio-loud population to the equivalent widths in the radio-quiet population. We have used the comparison between the LBQS and the PKS sample from Corbin's paper, and have assigned errors to his LBQS values using his sample size and the standard deviations we measure from the same sample. Our data are inadequate for deblending the feature on the blue wing of $\mathrm{C} \mathrm{III],} \mathrm{but} \mathrm{the} \mathrm{weakness} \mathrm{of} \mathrm{this} \mathrm{feature} \mathrm{means}$ that it does not invalidate the comparison with Sargent and Steidel's deblended data.

The first conclusion to be drawn from Table 3 is that all the measurements agree to within a standard deviation. Second, note that both Corbin and Steidel \& Sargent find excess emission in C IV; while it is not statistically significant in either paper individually, combining the data enhances the significance of the result. The combined data on C III] and $\mathrm{Mg}$ II confirm the similarity of these lines in radio-loud and quiet quasars, and tighten the limits on how much they can differ. Corbin confirms our detection of a marginal excess in the $\mathrm{Fe}$ II emission at around $2400 \AA$, but even with the combined data the excess is only significant at the $1.8 \sigma$ level.

The good agreement between different authors is not seen in the velocity width measurements. Corbin reports $\mathrm{C}$ IV velocity widths $30 \%$ larger in radio-loud quasars (the PKS sample) than in radio-quiet (the LBQS sample); the opposite sense to that reported here. However, his other comparison (BQS vs Corbin's observations) gives a contrasting result; marginally broader lines in the radio-quiet sample. The LBQS measurement in his first comparison gives a substantially lower width than our measurements of the same sample: the difference may result from his measuring the width not from the individual spectra, but from a composite spectrum.

A final caution: The majority of the radio-loud quasars in both Steidel \& Sargent and Corbin were selected by surveys at longer wavelengths and much lower sensitivity than the radio observations reported here. The differences may reflect a different balance between steep and flatspectrum sources, or some correlation with radio power.

\section{DISCUSSION}

\subsection{Broad Absorption Line Quasars}

A popular model for BALQSOs is that all radio-quiet and radio-moderate quasars have $\mathrm{BAL}$ clouds covering $\sim 10 \%$ of the sky as seen from the central engine. This naturally explains the essential similarity of the optical spectra of BALQSOs and normal quasars (PALBAL). To explain the abundance of BALQSOs amongst the radiomoderate population, however, stronger radio emission must be seen when observing on lines of sight that pass through the BAL clouds. This might occur if the radio emission came from a relativistic jet, and if the BAL clouds were concentrated near the jet axis. Note that this model does not purport to explain radio-loud quasars; they cannot be part of this unification scheme for radio-moderate and radio-quiet quasars.

How fast must the radio jet be to generate the necessary anisotropy? The bulk of the radio-quiet population lies at $\log _{10}(R) \sim-0.5$ (Fig. 1), and the radio-moderate quasars have $\log _{10}(R)$ as high as 0.6 . We therefore need a factor of $\sim 10$ variation in $R$ as a function of orientation. If the optical emission is isotropic, and the radio-flux comes from a tightly collimated two-sided jet, the radio-emitting material must be traveling with $v \sim 0.6 c(\gamma \sim 1.3)$ to generate the necessary anisotropy by relativistic beaming, assuming a radio spectral index $\alpha$ of -0.5 . The jet is thus only moderately relativistic.

As the jet is only moderately relativistic, it will beam the radio flux into a wide solid angle, so a large fraction of quasars will be radio-moderate. If the peak of the radioquiet population lies at around $\log _{10}(R) \sim-0.5$, as suggested by Fig. 1, then this represents the flux from quasars observed at $90^{\circ}$ to the jet axis. Given jets with $\gamma \sim 1.3$, and assuming that the optical radiation is isotropic, about $20 \%$ of quasars will have jets pointing near enough to the line of sight that they will appear radio moderate. Allowing for the sample completeness, $\sim 45$ quasars should have been observed with $0.2<\log _{10}(R)<1$, but only 10 were seen, a discrepancy significant at the $99.9 \%$ confidence level.

The simple beaming model thus significantly overpredicts the numbers of radio-moderate quasars. A less collimated beam would only increase the predicted fraction of radio-moderate quasars. This result, however, assumed that the optical radiation was isotropic. Any anisotropy in the optical radiation will bias an optically magnitudelimited sample such as the LBQS toward quasars with particular orientations. BAL clouds near the jet axis will diminish the optical flux, causing pole-on quasars to drop out of the survey. Typical BAL troughs only diminish the optical flux by $\sim 20 \%$, which given the steepness of the quasar luminosity function will cut their observed numbers by a factor of $\sim 2$ (Francis 1993). Furthermore, at redshifts below 1.7, the BAL absorption does not appear in the $B_{J}$ band, so over half the survey quasars are not affected. Thus at most, the BAL absorption will reduce the predicted numbers of radio-moderate quasars by $25 \%$, insufficient to bring the model into agreement with the observations.

If the rest-frame UV flux comes from an accretion disk, or from a jet, it will be significantly anisotropic, and quasars observed down the jet axis will have enhanced optical luminosities. This has two opposite effects on the predicted distribution of $R$. First, because the radio and optical fluxes are anisotropic in the same sense, the jet needs to be more strongly relativistic to give the same range in $R$. A more relativistic jet will beam its radio flux into a smaller solid angle, reducing the predicted numbers of radio-moderate quasars. On the other hand, quasars beamed toward us will be over-represented in the survey, due to their optical 
brightness. To evaluate the relative importance of these two opposing effects, we assume that the observed optical flux varies by a factor of three with orientation angle, the greatest anisotropy allowed by the equivalent-width distribution (Francis 1993). A radio-jet with $\gamma=1.7$ is now required to generate the observed range of $R$. This reduces the predicted number of radio-moderate quasars by a factor of two. However, the quasars viewed down the radio axis are three times brighter than those viewed from perpendicular to it, and will be over-represented in the sample by a factor of $\sim 50$ (Francis 1993), far outweighing the effects of the higher jet velocity. We therefore conclude that relativistic beaming cannot unify radio-quiet and radio-moderate quasars.

One alternative to the orientation model for BALQSOs is to say that they are in some intrinsic way different from other radio-quiet quasars. An orientation model, in which all radio-quiet quasars are BALQSOs when viewed from the right orientation, is still possible if we relax the assumption that all BALQSOs have identical BAL cloud covering factors. Radio moderate quasars have $\sim 10 \times$ the radio flux of radio-quiet quasars, and BALQSOs are $\sim 10 \times$ more common amongst them. If we allow radio-quiet quasars to have a factor of ten dispersion in their BAL cloud covering factors, and if the radio-flux is proportional to the $B A L$ cloud covering factor, radio-quiet quasars will have $\sim 5 \%$ covering factors of BAL clouds, while radiomoderate quasars will have covering factors as high as $50 \%$, as observed. The physical problem is now to explain why the covering factor of BAL clouds should be roughly proportional to the radio flux.

We conclude that models in which all radio-quiet and radio-moderate quasars have the same $B A L$ cloud covering factor cannot explain why radio-moderate quasars tend to be BALQSOs. Either BALQSOs are a quite separate subpopulation, or BAL cloud covering factors must vary between quasars, and the radio flux must be proportional to the BAL cloud covering factor.

\subsection{Narrow-line Quasars}

As shown in Sec. 3.2.3, the observed emission-line differences between radio-loud and radio-quiet quasars can be interpreted as resulting from an overabundance of narrowline quasars (NLQSOs) in the radio-loud population. If a NLQSO is defined as any quasar with a first component weight in the Francis et al. (1992) classification scheme of more than 2.5 , then $\sim 70 \%$ of radio-loud quasars are NLQSOs, as opposed to $\sim 12 \%$ of radio-quiet quasars.

NLQSOs are characterized by strong narrow ( FWHM $\sim 2500 \mathrm{~km} \mathrm{~s}^{-1}$ ) cores on their $\mathrm{C}$ IV and Lyman- $\alpha$ emission lines. Note that while these cores are narrow in comparison with typical broad emission lines, they are substantially broader than classical narrow emission lines such as [O III]. The line wings and other emission lines differ little between NLQSOs and normal quasars, and NLQSOs lie on the tail of the normal distribution of quasar spectral properties, rather than being an entirely distinct sub-class. Francis et al. (1992), Francis (1993), and Baldwin et al.
(1988) argue that NLQSOs cannot be normal quasars viewed from some special orientation.

NLQSOs may therefore have an intrinsic difference in their broad emission-line regions. The observations can be satisfied if, in addition to a perfectly normal broad-line region (BLR), these quasars have an additional set of emission-line clouds outside the normal BLR. These extra clouds would have roughly half the velocity dispersion of the normal BLR, a high density $\left(\rho>10^{11} \mathrm{~cm}^{-3}\right)$ to eliminate $\mathrm{C}$ III] emission, a low column density to prevent $\mathrm{x}$-ray excitation of $\mathrm{Mg}$ II and a moderate ionization parameter; high enough to excite $\mathrm{C}$ IV but not high enough to excite N v. Support for this model comes from the variability mapping observations of Clavel (1991), in which the emission-line cores are found to lag the emission-line wings.

The question remains: why are these excess BLR clouds found preferentially in radio-loud and radio-moderate quasars? The radio jets may generate these clouds, perhaps by interacting with the interstellar medium, or both the radioemission and the clouds may be caused by some underlying parameter such as host galaxy type or the specific angular momentum of the accreting material.

Can NLQSOs have broad absorption lines? As the BAL troughs absorb Lyman- $\alpha$ this is not straightforward to measure. However, at least one LBQS quasar, 1314+0116, is both a BALQSO (as defined by PALBAL) and shows strong narrow $\mathrm{C}$ IV emission. The similarity between the emission-line properties of BALQSOs and normal quasars found by PALBAL further argues that NLQSOs must occur amongst the BALQSO class with approximately normal frequency.

\subsection{Low Ionization Emission Lines}

This paper confirms the remarkable similarity between the $\mathrm{Mg}$ II and $\mathrm{CIII}$ ] emission-lines of radio-quiet and radio-loud quasars. The absence of measurable differences in these lines, compared to the strong differences found for Lyman- $\alpha$ and $\mathrm{C}$ IV, is evidence in favor of the twocomponent models of the broad-line region (e.g., CollinSouffrin et al. 1988), in which high and low ionization lines arise in different physical regions.

A caution: the quasars used to study $\mathrm{Mg}$ II and C III] have lower redshifts and luminosities than those used to study the high ionization lines. The difference in their behavior may thus be an evolutionary or luminosity effect. The emission-line properties of the LBQS as a whole do not, however, show any strong dependence on luminosity or redshift (Francis et al. 1992).

The similarity in the equivalent width of $\mathrm{Mg}$ II is particularly surprising in light of the observed high x-ray-tooptical flux ratios of radio-loud quasars (Lawson et al. 1992 and references therein). Radio-loud quasars typically have double the x-ray-to-optical flux ratios of their radioquiet counterparts, while their $\mathrm{Mg}$ II equivalent widths agree to within $10 \%$ (Table 3 ). While the $x$-ray observations are for much lower redshift, less luminous quasars 
than those in the LBQS, this result suggests that $\mathrm{x}$-ray heating is not a dominant process in creating lowionization lines.

\section{CONCLUSIONS}

The three major conclusions of this paper are:

(1) Radio-loud and radio-quiet quasars have remarkably similar low-ionization emission lines.

(2) The most radio-bright $5 \%$ of the radio-quiet population have spectral properties differing both from radioloud and radio-quiet quasars. Over $50 \%$ of these radio- moderate quasars have broad absorption lines, as compared with $\sim 5 \%$ of radio-quiet quasars and none of the radio-loud quasars.

(3) The majority of radio-loud quasars have strong narrow cores to their Lyman- $\alpha$ and $\mathrm{C}$ IV emission lines.

The LBQS is supported by National Science Foundation Grant No. AST 90-01181, for which we are grateful. Peter Strittmatter kindly made funds available for publication. We also wish to thank Craig Foltz and Paul Hewett for many helpful discussions. P.J.F. is supported by a SERC/ NATO advanced fellowship.

\section{REFERENCES}

Baldwin, J. A., McMahon, R., Hazard, C., \& Williams, R. E. 1988, ApJ, 327, 103

Baldwin, J. A., Wampler, E. J., \& Gaskell, C. M. 1989, ApJ, 338, 630

Bennett, C. L., Lawrence, C. R., Burke, B. F., Hewitt, J. N., \& Mahoney, J. 1986, ApJS, 61, 1

Boroson, T. A., \& Green, R. F. 1992, ApJS, 80, 109

Chaffee, F. H., Foltz, C. B., Hewett, P. C., Francis, P. J., Weymann, S. L.,

Morris, S. L., Anderson, S. F., \& MacAlpine, G. M. 1991, AJ, 102, 461

Clavel, J. 1991, in Variability of Active Galaxies, edited by W. J. Duschl,

S. J. Wagner, and M. Camenzind (Springer, Berlin), p. 31

Collin-Souffrin, S. Dyson, J. E., McDowell, J. C., \& Perry, J. J. 1988, MNRAS, 232, 539

Corbin, M. R. 1991, ApJ, 375, 503

Corbin, M. R. 1992, ApJ, 391, 577

Feigelson, E. D., \& Nelson, P. I. 1985, 293, 192

Foltz, C. B., Chaffee, F. H., Hewett, P. C., MacAlpine, G. M., Turnshek,

D. A., Weymann, R. J., \& Anderson, S. F. 1987, AJ, 94, 1423

Foltz, C. B., Chaffee, F. H., Hewett, P. C., Weymann, R. J., Anderson, S.

F., \& MacAlpine, G. M. 1989, AJ, 98, 1959

Foltz, C. B., Weymann, R. J., Peterson, B. M., Sun, L., Malkan, M. A., \& Chaffee, F. H. 1986, ApJ, 307, 504

Francis, P. J. 1991, thesis, University of Cambridge

Francis, P. J. 1993, ApJ (in press)

Francis, P. J., Hewett, P. C. Foltz, C. B., \& Chaffee, F. H. 1992, ApJ, 398, 476
Francis, P. J., Hewett, P. C., Foltz, C. B., Chaffee, F. H., Weymann, R. J., \& Morris, S. L. 1991, ApJ, 373, 465

Hewett, P. C., Foltz, C. B., Chaffee, F. H., Francis, P. J., Weymann, R. J., Morris, S. L., Anderson, S. F., \& MacAlpine, G. M. 1991, AJ, 101, 1121

Hooper, E. J., Impey, C. D., Foltz, C. B., \& Hewett, P. C. 1993, in preparation

Kellerman, K., Sramek, R., Schmidt, M., Shaffer, D., \& Green, R. 1989, AJ, 98, 1195

Lawson, A. J., Turner, M. J. L., Williams, O. R., Stewart, G. C., \& Saxton, R. D. 1992, MNRAS, 258, 743

Morris, S. L., Weymann, R. J., Anderson, S. F., Hewett, P. C., Foltz, C. B., Chaffee, F. H., Francis, P. J., \& MacAlpine, G. M. 1991, AJ, 102, 1627

Sprayberry, D., \& Foltz, C. B. 1992, ApJ, 390, 39

Steidel, C. C., \& Sargent, W. L. W. 1991, ApJ, 382, 433

Stocke, J. T., Morris, S. L., Weymann, R. J., \& Foltz, C. B. 1992, ApJ, 396,487

Veron-Cetty, M. P., \& Veron, P. 1987, A Catalogue of Quasars and Active Galactic Nuclei, 3rd ed. (ESO, Munich)

Visnovsky, K. L., Impey, C. D., Foltz, C. B., Hewett, P. C., Weymann, R. J., \& Morris, S. L. 1992, ApJ, 391, 560

Weymann, R. J., Morris, S. L., Foltz, C. B., \& Hewett, P. C. 1991, ApJ, 373, 23 (PALBAL)

Wills, B. J., Netzer, H., \& Wills, D. 1985, ApJ, 288, 94 